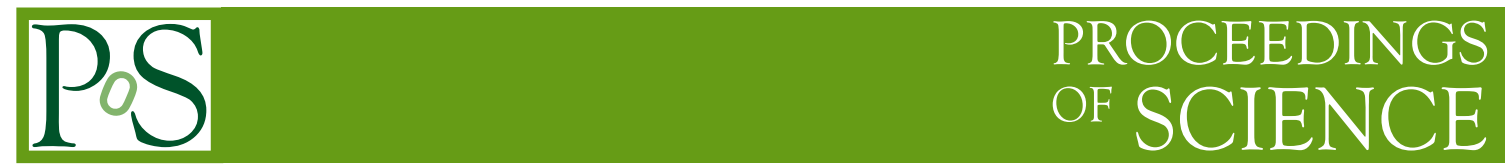

\title{
Search for Higgs bosons in CMS experiment
}

\author{
Roberta Volpe on behalf of the CMS Collaboration* \\ National Central University, Taiwan \\ E-mail: roberta.volpe@cern.ch
}

The recent Higgs results from CMS based on the data collected in 2011 and 2012 at 7 and $8 \mathrm{TeV}$ centre-of-mass energy, corresponding to integrated luminosities of 5 and $20 \mathrm{fb}^{-1}$, are described. The observed new boson with a measured mass of about $125.7 \pm 0.3$ (stat.) \pm 0.3 (syst.) $\mathrm{GeV}$ is found to be consistent with the Standard Model Higgs boson in all investigated properties.

The XXI International Workshop High Energy Physics and Quantum Field Theory,

June 23 - June 30, 2013

Saint Petersburg Area, Russia

${ }^{*}$ Speaker. 


\section{Introduction}

In the Standard Model [1][2] (SM) one scalar Higgs boson is predicted to exist [3] [4]. In July 2012, combining the $7 \mathrm{TeV}$ data with the first $5 \mathrm{fb}^{-1}$ collected at $8 \mathrm{TeV}$ centre-of-mass (CM) energy, ATLAS and CMS discovered a new boson with a mass around $126 \mathrm{GeV}$ [5][6]. CMS [12] has now analyzed the full dataset collected in 2012 that amounts to $19.6 \mathrm{fb}^{-1}$.

There are four main Higgs boson production modes in pp collisions at $\sqrt{s}=7,8 \mathrm{TeV}$. The gluon-gluon fusion production mode $(\mathrm{ggH})$ has the largest cross section, followed in turn by vector boson fusion $(V B F)$, associated $\mathrm{WH}$ and $\mathrm{ZH}$ production $(V H)$, and production in association with top quarks $(t t H)$. The cross sections for the Higgs boson production modes and the decay branching fractions, together with their uncertainties, are taken from Refs. [7], [8], [9] and are derived from Refs. [10][11]. The total cross section at $\sqrt{s}=7(8) \mathrm{TeV}$ varies from 17.8 (22.7) to 17.0 (21.6) pb for a Higgs boson mass range from 124 to $127 \mathrm{GeV}$. The main results of this report are obtained by studying the Higgs decay channels: $\gamma \gamma, Z Z, W W, \tau \tau$ and $b \bar{b}$. The several analyses using such decays have been combined to get more sensible estimations and tests for the properties of the new particle. Tab. 1 lists these analyses by specifying which was the production they were aimed to, the exclusive final states exploited, and the integrated luminosity used.

\begin{tabular}{|l|c|l|c|c|}
\hline H decay & Prod.Tag & Exclusive final states & $7 \mathrm{TeV}$ & $8 \mathrm{TeV}$ \\
\hline$\gamma \gamma$ & untagged & $\gamma \gamma$ & 5.1 & 19.6 \\
$\gamma \gamma$ & VBF-tagged & $\gamma \gamma+(j j)_{V B F}$ & 5.1 & 19.6 \\
$\gamma \gamma$ & VH-tagged & $\gamma \gamma+(e, \mu, \mathrm{MET})$ & - & 19.6 \\
\hline$Z Z \rightarrow 4 l$ & $N_{\text {jet }}<2$ & $4 e, 4 \mu, 2 e 2 \mu$ & 5.1 & 19.6 \\
$Z Z \rightarrow 4 l$ & $N_{\text {jet }} \geq 2$ & $4 e, 4 \mu, 2 e 2 \mu$ & 5.1 & 19.6 \\
\hline$W W \rightarrow l v l v$ & $0 / 1$ Jets & (DF or SF dileptons $) \times(0$ or 1 jet $)$ & 4.9 & 19.5 \\
$W W \rightarrow l v l v$ & VBF-tag & $l v l v+(j j)_{V B F}$ & 4.9 & 12.1 \\
$W W \rightarrow l v l v$ & WH-tag & $3 l 3 v$ & 4.9 & 19.5 \\
\hline$\tau \tau$ & $0 / 1$ jet & $\left(e \tau_{h}, \mu \tau_{h}, e \mu, \mu \mu\right) \times\left(\right.$ low or high $\left.p_{T}^{\tau}\right)$ & 4.9 & 19.6 \\
$\tau \tau$ & 1 -jet & $\tau_{h} \tau_{h}$ & 4.9 & 19.6 \\
$\tau \tau$ & VBF-tag & $\left(e \tau_{h}, \mu \tau_{h}, e \mu, \mu \mu, \tau_{h} \tau_{h}\right)+(j j)_{V B F}$ & 4.9 & 19.6 \\
$\tau \tau$ & ZH-tag & $(e e, \mu \mu) \times\left(\tau_{h} \tau_{h}, e \tau_{h}, \mu \tau_{h}, e \mu\right)$ & 5.0 & 19.5 \\
$\tau \tau$ & WH-tag & $\tau_{h} \mu \mu, \tau_{h} e \mu, e \tau_{h} \tau_{h}$ & 5.0 & 19.5 \\
\hline$b b$ & VH-tag & $(v v, e e, \mu \mu, e v, \mu v$ with 2 b-jets $) \times(3$ kin. cat. $)$ & 5.0 & 12.1 \\
$b b$ & ttH-tag & $(l$ with 4,5, or 6 jets $) \times(2$ kin. cat $)$ & 5.0 & 5.1 \\
\hline
\end{tabular}

Table 1: List of the analyses which have been combined to get the main results of this report. Notation: $(j j)_{V B F}$ stands for a jet pair consistent with the VBF topology (VBF-tag); $S F$ are same flavour dileptons, $D F$ are different flavour dileptons, kin.cat means other categories based on kinematic variables.

This document is organized as in the following. The Sec. 2 is dedicated to a brief description of the CMS detector. Sec. 3 summarize the bases of statistical treatment of data, which have been used for both the single channel and the combination of several channels. Sec.4 describes in summary the selection and the strategy of the main analyses and Sec.5 reports the results which have been obtained from the combination of those. In Sec. 6 a brief overview of the results obtained with other 
analyses, not included in the combination, is presented.

\section{CMS Detector}

The central feature of the CMS detector [12] is a superconducting solenoid $13 \mathrm{~m}$ long, with an internal diameter of $6 \mathrm{~m}$. The solenoid generates a uniform 3.8 T magnetic field along the axis of the LHC beams. Within the superconducting solenoid volume are a silicon pixel and strip tracker, a lead tungstate crystal electromagnetic calorimeter, and a brass/scintillator hadron calorimeter. Muons are identified and measured in gas-ionisation detectors embedded in the outer steel magnetic flux return yoke of the solenoid. The detector is subdivided into a cylindrical barrel and endcap disks on each side of the interaction point. Calorimeters in the forward and backward directions complement the coverage provided by the barrel and endcap detectors.

\section{Statistical treatment of data}

The overall statistical methodology used both for the single decay channel and the combination was developed by the ATLAS and CMS Collaborations in the context of the LHC Higgs Combination Group. The chosen test statistic $q$, used to determine how signal- or background-like the data are, is based on the profile likelihood ratio. Systematic uncertainties are incorporated in the analysis via nuisance parameters and are treated according to the frequentist paradigm. The description of the general methodology can be found in Refs. [13][14]. Below a concise definitions of statistical quantities used for characterizing the outcome of the search is given. Results presented in this document are obtained using asymptotic formulae [15], including a few updates recently introduced in the RooStats package [16].

To quantify the presence of an excess of events over what is expected for the background, we use the test statistics where the likelihood appearing in the numerator is for the background only hypothesis:

$$
q_{0}=-2 \ln \frac{\mathscr{L}\left(o b s \mid b, \hat{\theta}_{0}\right)}{\mathscr{L}(o b s \mid \hat{\mu} \cdot s+b, \hat{\theta})}
$$

where $s$ stands for the signal expected under the SM Higgs hypothesis, $\mu$ is a signal strength modifier introduced to accommodate deviations from SM Higgs predictions, $b$ stands for backgrounds, and $q$ are nuisance parameters describing systematic uncertainties. The value $\hat{\theta}_{0}$ maximises the likelihood in the numerator under the background-only hypothesis $(\mu=0)$, while $\hat{\mu}$ and $\hat{\theta}$ define the point at which the likelihood reaches its global maximum. The probability $p_{0}$, henceforth referred to as the local $p$-value, is defined as the probability to obtain a value $q_{0}$ at least as large as the one observed in data, $q_{0}^{o b s}$, under the background-only $(b)$ hypothesis:

$$
p_{0}=P\left(q_{0} \geq q_{0}^{o b s} \mid b\right)
$$

The local significance $Z$ of a signal-like excess is then computed from the following equation, using the one-sided Gaussian tail convention:

$$
p_{0}=\int \frac{1}{\sqrt{2 \pi}} \exp \left(-x^{2} / 2\right) d x
$$


Signal model parameters $a$ (the signal strength modifier $\mu$ can be one of them) are evaluated from a scan of the profile likelihood ratio $q(a)$ :

$$
q(a)=-2 \ln \frac{\mathscr{L}\left(o b s \mid s(a)+b, \hat{\theta}_{0}\right)}{\mathscr{L}(o b s \mid s(\hat{a})+b, \hat{\theta})}
$$

The parameters $\hat{a}$ and $\hat{\theta}$ that maximise the likelihood, $\mathscr{L}($ obs $\mid s(\hat{a})+b, \hat{\theta})=\mathscr{L}_{\max }$, are called the best-fit set.

\section{The analyses used in the combination}

As anticipated in Sec.1, the results of the most significant analyses, listed in Tab.1 have been combined to study the properties of the newly discovered particle. Below a quick overview of these analyses is given. A detailed description of the reconstruction and identification of the physics objects, and of the analysis description, can be found in the cited references.

\section{1 $H \rightarrow \gamma \gamma$}

In the $H \rightarrow \gamma \gamma$ analysis [18], we search for a narrow mass peak over a smoothly falling background due to QCD prompt diphoton production and to events with at least one jet misidentified as an isolated photon. The event sample is split into several mutually exclusive classes: $(i)$ diphoton events without any tagging object (untagged), (ii) diphoton events with one forward and one backward jet, consistent with the VBF topology, and (iii) diphoton events with a high- $p_{T}$ muon or electron or large $E_{\text {miss }}^{T}$, consistent with associated production with a $Z$ or $W$ boson. The $E_{\text {miss }}^{T}$ variable is defined as the modulus of the vector $\vec{E}_{\text {miss }}^{T}$ computed as the negative of the vector sum of the transverse momenta of all reconstructed objects in the detector (leptons, photons, and charged/neutral hadrons) which are not found to arise from additional interactions. This classification is motivated by the better signal-to-background ratio in the last two types of event classes. For the $8 \mathrm{TeV}$ data, the dijet class is split into two sub-classes according to a multivariate (MVA) classifier. The untagged events contain over $99 \%$ of the data. They are subdivided into four sub-classes based on the output of a MVA classifier that assigns a high score to signal-like events, based on combination of (a) an event-by-event estimate of the diphoton mass resolution, (b) a photon identification score for each photon, and (c) kinematic information about the photons and the diphoton system. The photon identification score is obtained from another MVA classifier that uses shower shape information and isolation variables to separate prompt photons from those arising from jets. The background in the signal region is estimated from fits to each of the observed diphoton mass distributions in data. This analysis has also been performed using a cut-based approach described in [18]. The exclusion limit and the observed local $p$-value, calculated with the procedure described in Sec.3, obtained with the main analysis, are shown in Fig.1.

\section{2 $H \rightarrow Z Z$}

In the $H \rightarrow Z Z^{(*)} 4 l$ channel [19], we search for a four-lepton mass peak over a small continuum background. To further separate signal and background, we use a discriminant calculated from the leading order matrix elements for signal and background to form an event with the observed kinematics (the masses of the dilepton pairs and five angles fully defining a four-lepton configuration 

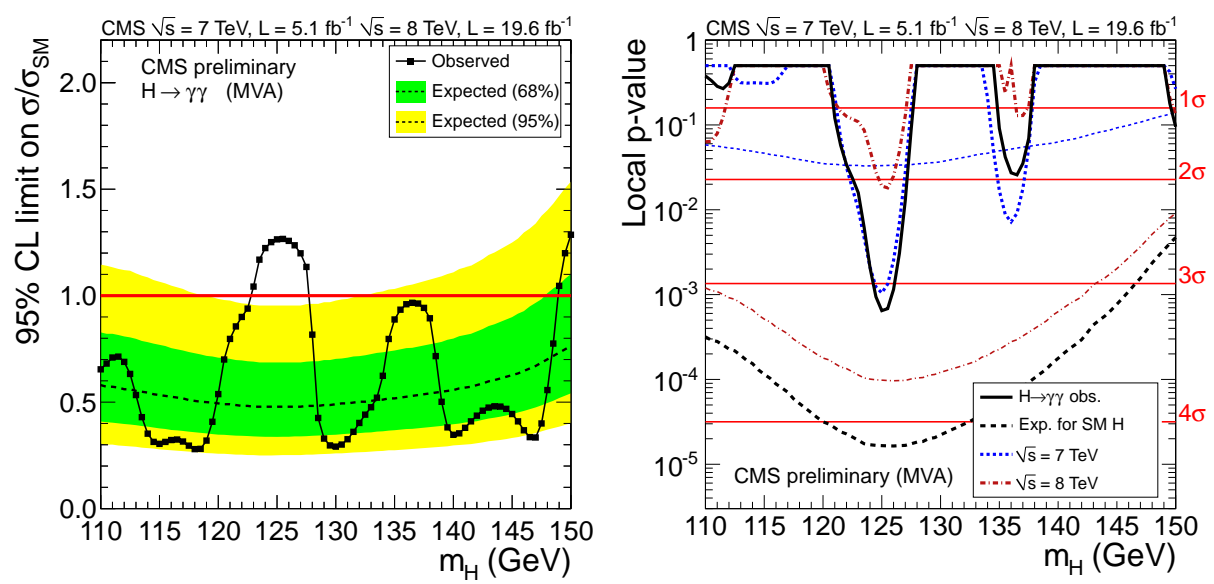

Figure 1: Left: the exclusion limit on the cross section of a SM Higgs boson decaying into two photons as a function of the boson mass hypothesis relative to the SM cross section. Right: Observed local $p$-values as a function of $m_{H}$.

in their centre-of-mass frame). The $4 e, 4 \mu$ and $2 e 2 \mu$ sub-channels are analysed separately since there are differences in the four-lepton mass resolutions and the background rates arising from jets misidentified as leptons. The dominant irreducible background in this channel is from non-resonant $Z Z$ production with both $Z$ bosons decaying to $2 e, 2 \mu$, or $2 \tau$ (with the taus decaying leptonically) and is estimated from simulation. The smaller reducible backgrounds from $Z \rightarrow b b, \tau \tau$, and from $Z+$ jets with jets misidentified as leptons are estimated from data. Fig.2 shows the distribution of four-lepton reconstructed mass for data and the estimated background, both for the $4 l$ and the $2 l 2 \tau$ channels. In the four leptons channels, the fully reconstructed decay and properties of the Higgs
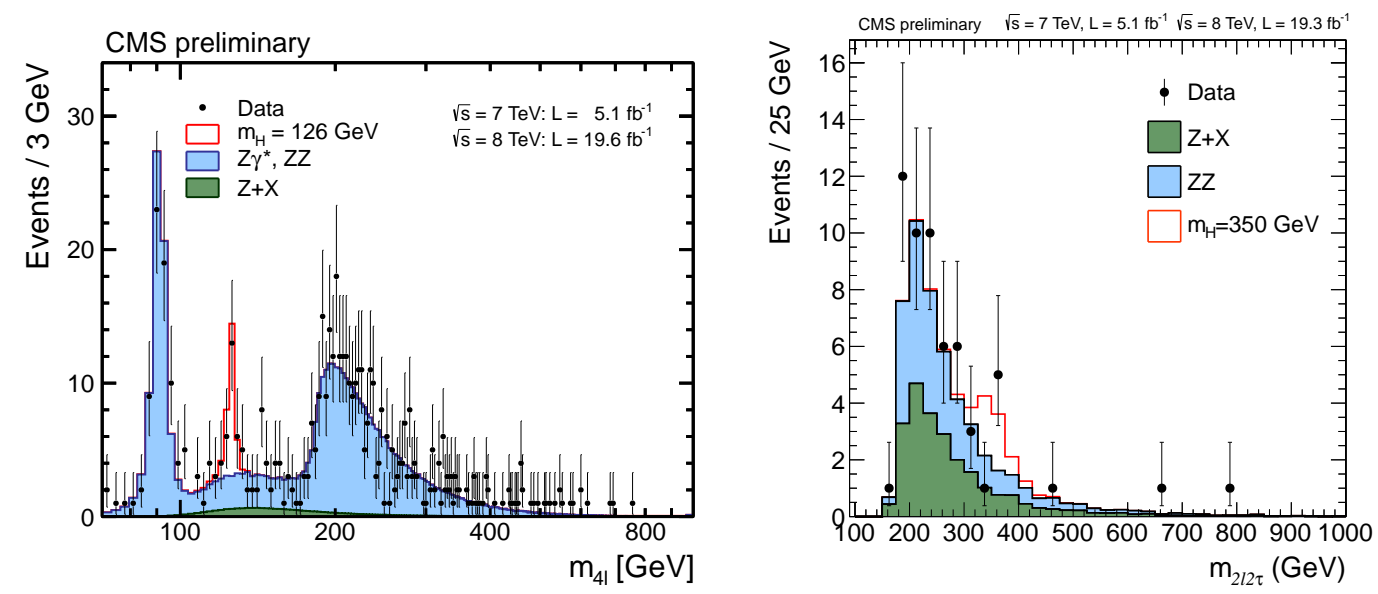

Figure 2: Distribution of the four-lepton reconstructed mass in the full mass range for the sum of the $4 e, 4 \mu$, and $2 e 2 \mu$ channels (left), and for the sum over all $l^{+} l^{-} \tau^{+} \tau^{-}$channels (right)

boson are exploited to increase the sensitivity of the analysis. Since the Higgs boson is spinless, the angular distribution of its decay products is independent of the production mechanism. These 
angular variables, together with the invariant masses of the two $Z$ bosons, are used to construct a kinematic discriminant $\left(K_{D}\right)$. The distributions for data, signal and background of the 2D plane $\left(m_{4 l}, K_{D}\right)$ are shown in Fig.3. To increase the sensitivity to the production mechanism, the event
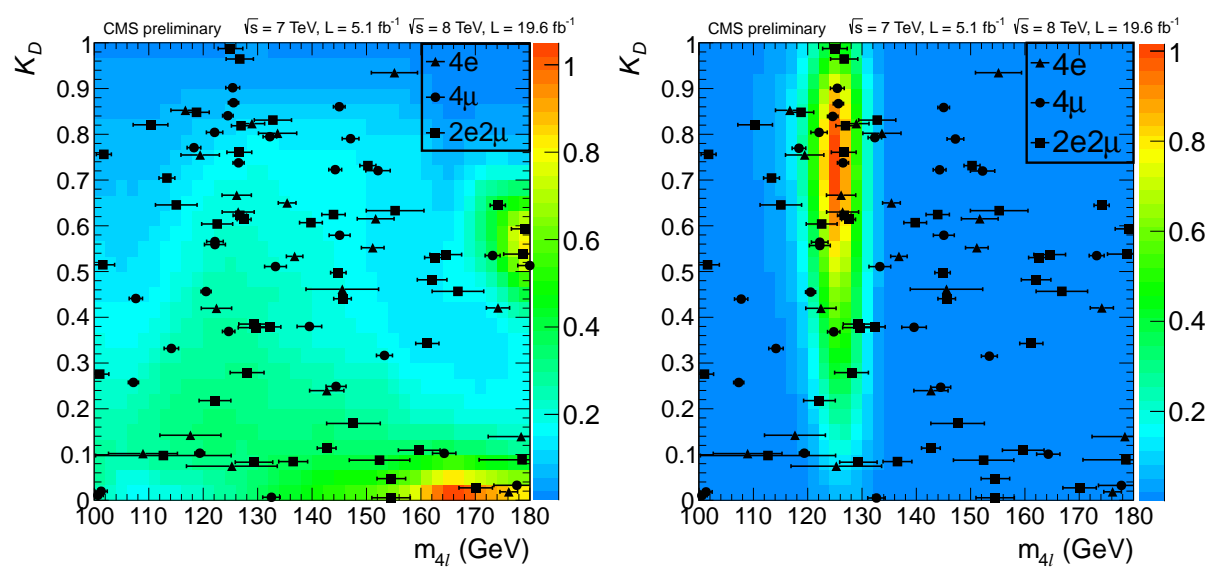

Figure 3: Distribution of the kinematic discriminant $K_{D}$ versus the four-lepton reconstructed mass $m_{4 l}$ with contours shown for the expected relative density of background events (left) and signal events for $\mathrm{mH}=126$ $\mathrm{GeV}$ (right). The points show the data with measured invariant mass uncertainties as horizontal bars.

sample is split into two categories based on jet multiplicity: $i$ ) events with fewer than two jets, and ii) events with at least two jets. In the first category, the $p_{T}(4 l) / m(4 l)$ ratio is used to discriminate $\mathrm{VBF}$ and $\mathrm{VH}$ production from gluon-gluon fusion. In the second category a linear discriminant $\left(V_{D}\right)$, formed from two VBF sensitive variables (the difference in pseudo-rapidity between the 2 leading jets and their invariant mass), is used to separate the VBF and gluon-gluon fusion processes. Fig. 4 shows the results for $\mu=\sigma / \sigma_{S M}$ and the local $p$-values obtained with this channel.
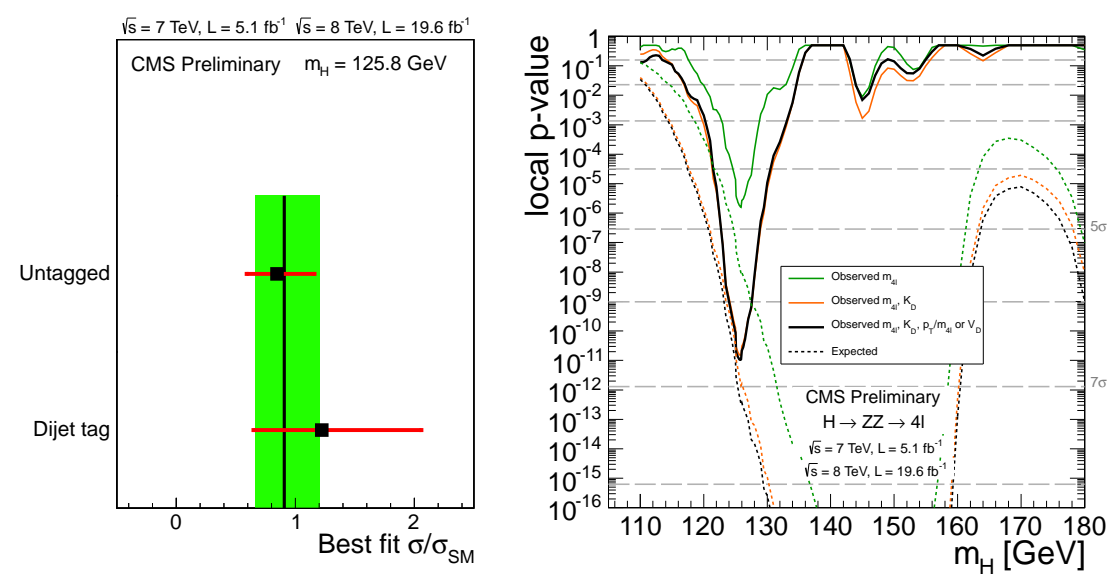

Figure 4: Left: Values of $\mu=\sigma / \sigma_{S M}$ for the two categories. Right: Significance of the local excess with respect to the SM background expectation as a function of the Higgs boson mass for the 1D $\left(m_{4 l}\right), 2 \mathrm{D}$ $\left(m_{4 l}, K_{D}\right)$ and $3 \mathrm{D}\left(m_{4 l}, K_{D}, p_{T} / m_{4 l}\right.$ or $\left.V_{D}\right)$ models. 


\section{$4.3 H \rightarrow W W$}

In the $H \rightarrow W W^{(*)} \rightarrow 2 l 2 v$ channel [20] [21], we search for an excess of events with two leptons of opposite charge, large $E_{\text {miss }}^{T}$, and up to two jets. Events are divided into six categories, with different background compositions and signal-to-background ratios. For events with no jets, the main background stems from non-resonant WW production; for events with one jet, the dominant backgrounds are from $W W$ and top-quark production. The events are split into sameflavour and different-flavour dilepton sub-channels, since the background from Drell-Yan production $\left(q q \rightarrow \gamma^{*} / Z^{(*)} \rightarrow l l\right)$ is much larger for the same-flavour dilepton events. The two-jet category is optimised to take advantage of the VBF Higgs boson production signature. The main background in this channel is from top-quark production. The $2 \mathrm{D}$ distribution of events in the $\left(m_{l l}, m_{T}\right)$ plane is used for different-flavour dilepton channels with zero and one jet; $m_{l l}$ is the invariant mass of the dilepton pair, and $m_{T}$ is the transverse mass reconstructed from the transverse momentum of the dilepton pair and the vector $E_{\text {miss }}^{T}$. All background rates, except for very small contributions from $W Z, Z Z$, and $W \gamma$, are evaluated from data. In the $W H \rightarrow W W W \rightarrow 3 l 3 v$ channel [22], we search for an excess of events with three leptons, electrons or muons, large missing transverse energy, and low hadronic activity. The dominant background is from $W Z \rightarrow 3 v$ production, which is largely reduced by requiring that all sameflavour, oppositely charged lepton pairs have a dilepton mass away from the $\mathrm{Z}$ boson mass. In addition, oppositely charged leptons are required not to be back-to-back. The background processes with jets misidentified as leptons, e.g. $Z+$ jets and top production, as well as the $W Z \rightarrow 3 l 3 v$ background are estimated from data. The small contribution from the $Z Z \rightarrow 4 l$ process with one of the leptons having escaped detection is estimated using simulated samples. To give an idea of the background composition, the distributions of azimuthal angle difference between two selected leptons and the dilepton mass of the two selected leptons are shown in Fig.5.
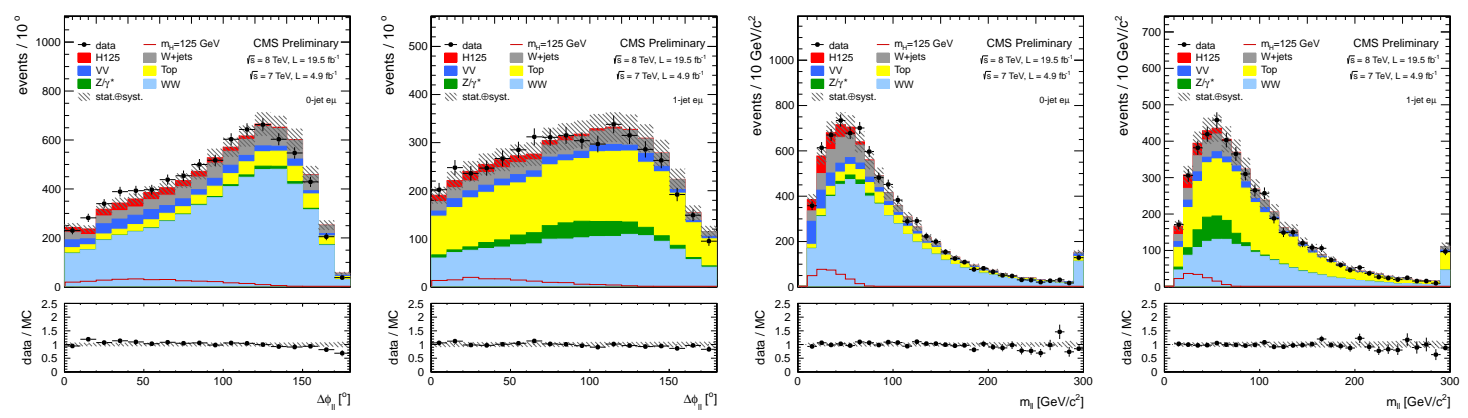

Figure 5: Distributions of the azimuthal angle difference between two selected leptons and the dilepton mass of the two selected leptons in the 0-jet and 1-jet categories in the different flavor final state, for data (points with error bars), for the main backgrounds (stacked histograms), and for a SM Higgs boson signal with $\mathrm{mH}=125 \mathrm{GeV}$ (superimposed histogram)

Fig.6 shows the Expected and observed 95\% CL upper limits on the cross section times branching fraction, $\sigma_{H} \times B R\left(H \rightarrow W^{+} W^{-}\right)$, relative to the SM Higgs expectation and the best fit value of $\sigma / \sigma_{S M}$, for each Higgs mass hypothesis, for the combined $7+8 \mathrm{TeV}$ analysis. 

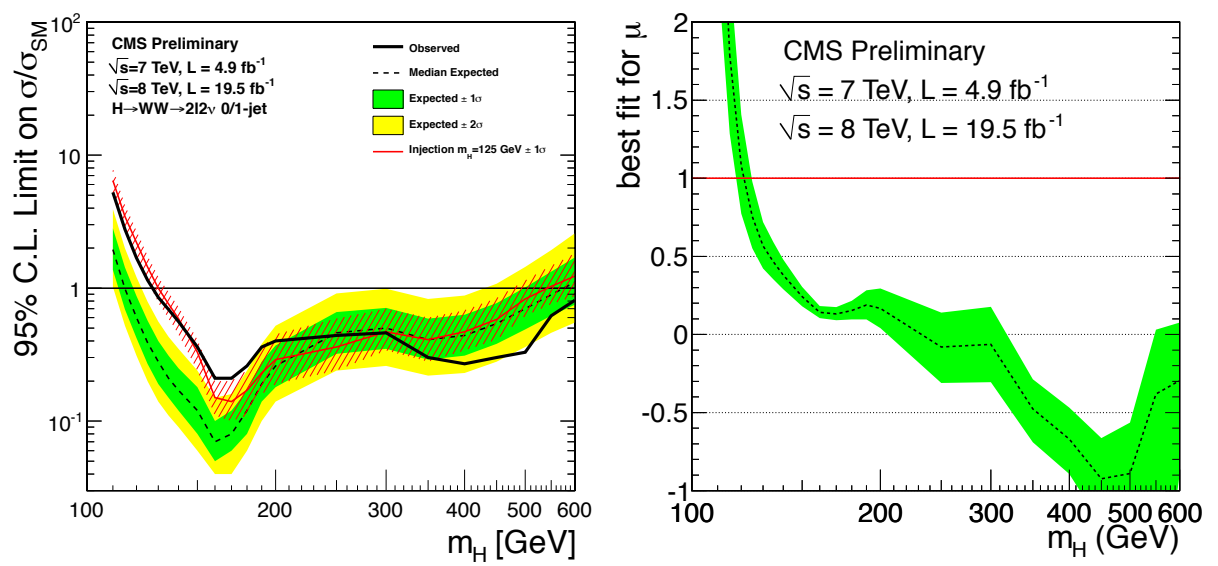

Figure 6: Left: Expected and observed 95\% CL upper limits on the cross section times branching fraction, $\sigma_{H} \times B R\left(H \rightarrow W^{+} W^{-}\right)$, relative to the SM Higgs expectation. Right: The best fit value of $\sigma / \sigma_{S M}$ for each Higgs mass hypothesis for the combined $7+8 \mathrm{TeV}$ analysis.

\section{4 $H \rightarrow \tau \tau$}

The $H \rightarrow \tau \tau$ search [23] is performed using the final-state signatures $e \mu, \mu \mu, e \tau_{h}, \mu \tau_{h}, \tau_{h} \tau_{h}$, where electrons and muons arise from leptonic $\tau$-decays and $\tau_{h}$ denotes a $\tau$ lepton decaying hadronically. Each of these categories is further divided into two exclusive sub-categories based on the number and the type of the jets in the event: (i) events with one forward and one backward jet, consistent with the VBF topology, (ii) events with at least one high $p_{T}$ hadronic jet but not selected in the previous category. The second category is further split in two bins of reconstructed $\tau$ lepton $p_{T}$. In each of these categories, we search for a broad excess in the reconstructed $\tau \tau$ mass distribution. The zero-jet category is used to constrain background normalisations, identification efficiencies, and energy scales. The main irreducible background, $Z \rightarrow \tau \tau$ production, and the largest reducible backgrounds $(W+$ jets, multijet production, $Z \rightarrow e e$ ) are evaluated from various control samples in data. The search for $H \rightarrow \tau \tau$ decays produced in association with a $W$ or $Z$ boson is conducted in events with three or four leptons in the final state [24]. The $W H$ analysis selects events which have electrons or muons of the same charge and one or two hadronically-decaying tau leptons: $e m \tau_{h}, \mu^{+} \mu^{+} \tau_{h}, e \tau_{h} \tau_{h}$, and $\mu \tau_{h} \tau_{h}$. The $\mathrm{ZH}$ analysis is performed in events with an identified $Z \rightarrow e e$ or $Z \rightarrow \mu \mu$ decay and a a Higgs boson candidate with one of the following final states: $e \mu, e \tau_{h}, \mu \tau_{h}, o r \tau_{h} \tau_{h}$. The main irreducible backgrounds to the $W H$ and $Z H$ searches are $W Z$ and $Z Z$ diboson events, respectively. The irreducible backgrounds are estimated using simulation, corrected by control samples in data. The reducible backgrounds in both analyses are $W, Z$, and $t \bar{t}$ events with at least one quark or gluon jet misidentified as an isolated $e, \mu$, or $\tau_{h}$. These backgrounds are estimated solely from data by measuring the probability for jets to be misidentified as isolated leptons in background-enriched control regions, and weighting the selected events which fail the lepton requirements by the misidentification probability. This estimated background is shown in Fig.7 (left) compared to the data for the distribution of the $\tau \tau$ invariant mass.

The combined observed 95\% CL upper limit on the signal strength parameter $\mu=\sigma / \sigma_{S M}$, together with the expected limit obtained in the background hypothesis is shown in Fig.7 (right). 

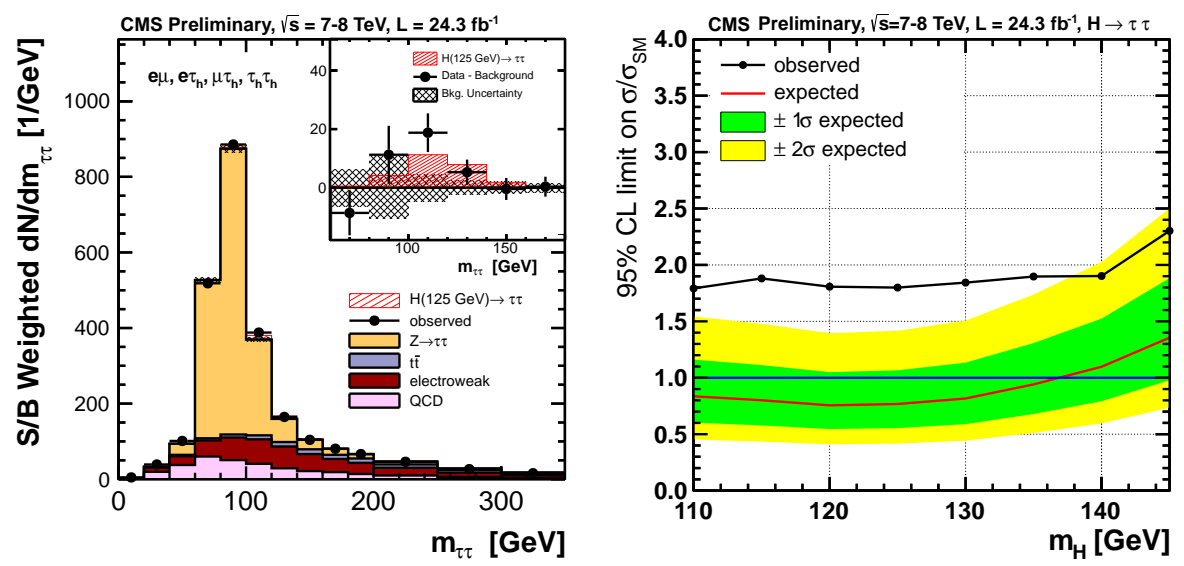

Figure 7: Left: Combined observed and expected $m_{\tau \tau}$ distributions for the $\mu \tau_{h}, e \tau_{h}, e \mu$ and $\tau_{h} \tau_{h}$ channels. The distributions obtained in each category of each channel are weighted by the ratio between the expected signal and background yields in the category Right: Combined observed 95\% CL upper limit on the signal strength parameter $\mu=\sigma / \sigma_{S M}$, together with the expected limit obtained in the background hypothesis.

\section{$4.5 H \rightarrow b b$}

The $H \rightarrow b b$ search [25] concentrates on Higgs boson production in association with a $W$ or $Z$ boson, in which the focus is on the following decay modes: $W \rightarrow e v / \mu v$ and $Z \rightarrow e e / \mu \mu / \nu v$. The $Z \rightarrow v v$ decay is identified by requiring large missing transverse energy. The Higgs boson candidate is reconstructed by requiring two $b$-tagged jets. The search is divided into events where the vector bosons have medium or large transverse momentum and recoil away from the candidate Higgs boson. An MVA regression to better estimate the $b$-jet $p_{T}$ is trained on jets in simulated signal events and achieves a final dijet mass resolution of $8-9 \%$ for $m_{H}=125 \mathrm{GeV}$. The performance of the regression algorithm is checked in data using $W / Z+$ jets and $t \bar{t}$ events. Events with higher transverse momentum bosons have smaller backgrounds and a better dijet mass resolution. In the $8 \mathrm{TeV}$ analysis, for all final states except $Z \rightarrow l l$, the large transverse momentum events are further divided depending on whether they satisfy tight or loose $b$-tagging requirements. An MVA classifier, trained for several different values of the Higgs boson mass, is used to separate signal and background events. The rates of the main backgrounds, consisting of $W / Z+$ jets and top-quark events, are derived from signal-depleted control samples in data. The $W Z$ and $Z Z$ backgrounds with a $Z$ boson decaying to a pair of $b$-quarks, as well as the single-top background, are estimated from simulation. The MVA classifier output distribution is used as the final discriminant in the limit setting. The upper limit obtained is shown in Fig.8 (left). The search for $H \rightarrow b \bar{b}$ decays is also performed using events where the Higgs boson is produced in association with a top-quark pair [26]. This analysis uses events where the top-quark pair decays to either the lepton-plus-jets $(t \bar{t} \rightarrow l v j j b b)$ or dilepton $(t \rightarrow t \rightarrow l v v b b)$ final state. The major background in this search is toppair production accompanied by extra jets. We use a MVA to discriminate between background and signal events; information related to object kinematics, event shape, and the discriminant output from the b-tagging algorithm is used. The rates of background processes are estimated from theoretical expectations, and are further constrained through the inclusion of background enriched 
samples in the extraction of the final limit. The upper limit for the $t \bar{t} H$ channel is shown in Fig.8 (right).
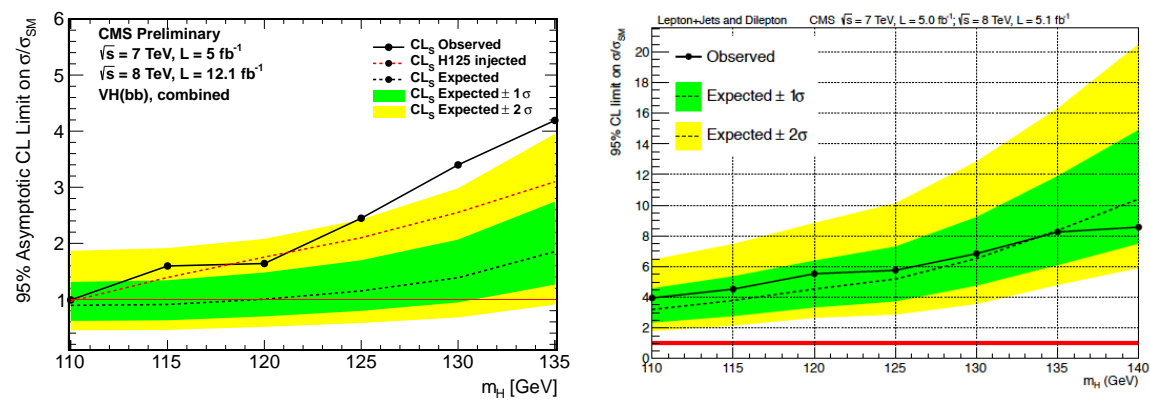

Figure 8: Expected and observed upper limit as a function of the Higgs boson mass for the $H \rightarrow b \bar{b}$ decay channel, for analyses targeting the $V H$ (left) and $t \bar{t} H$ (right) production modes.

\section{Results from the combination}

The combination of the Higgs boson searches requires simultaneous analysis of the data selected by all individual analyses, accounting for all statistical and systematic uncertainties and their correlations. All the results, can be found with a more detailed description in [17].

\subsection{Significance and mass measurement}

Table 9 (left) summarizes the median expected and observed local significance for a SM Higgs boson mass hypothesis of $125.7 \mathrm{GeV}$ from the individual decay modes. This value of the mass is the measured one. The expected significance is evaluated assuming the expected background and signal rates.

The $Z Z$ and $\gamma \gamma$ channels, that have excellent mass resolution, are used to estimate the mass of the boson. To extract the value of $m_{X}$ in a model-independent way, the signal strength modifiers for the $g g \rightarrow H \rightarrow \gamma \gamma, \mathrm{VBF}+\mathrm{VH}$ with $H \rightarrow \gamma \gamma$, and $H \rightarrow \mathrm{ZZ} \rightarrow 4 l$ processes are assumed to be independent and, thus, not tied to the SM expectation. The result is: $m_{X}=125 . \pm 0.3$ (stat.) \pm 0.3 (syst.) $\mathrm{GeV}$.

\subsection{Compatibility with the SM Higgs boson hypotesis}

The compatibility tests performed with this set of data show the consistency of the observations with the expectations for the SM Higgs boson.

\subsubsection{Signal strength}

The best fit value for the common signal strength modifier $\hat{\mu}=\hat{\sigma} / \sigma_{S M}$, obtained in the combination of all the search channels, is $\mu$, the values obtained for each channel are shown in Fig.10 (left). The relative strengths of the couplings to the vector bosons and top quarks have been tested by using a combination of the channels, explicitely targeting different production modes. 


\begin{tabular}{|l|c|c|}
\hline Decay mode & Exp. $(\sigma)$ & Obs. $(\sigma)$ \\
\hline$\gamma \gamma$ & 3.9 & 3.2 \\
$Z Z \rightarrow 4 l$ & 7.1 & 6.7 \\
$W W \rightarrow l v l v$ & 5.3 & 3.9 \\
$\tau \tau$ & 2.6 & 2.8 \\
$b b$ & 2.2 & 2.0 \\
\hline
\end{tabular}

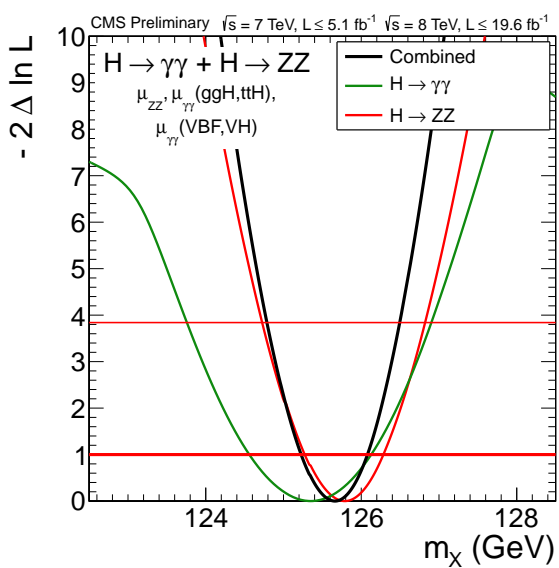

Figure 9: Left: The median expected and the observed significances of the excesses in the combination of the individual decay modes for a SM Higgs boson mass hypotesis of $125.7 \mathrm{GeV}$. Right: 1D-scan of the test statistic $q\left(m_{X}\right)=-2 \Delta \ln L$ versus the boson mass $\mathrm{mX}$ for the $\gamma \gamma$ and $4 l$ final states separately and for their combination
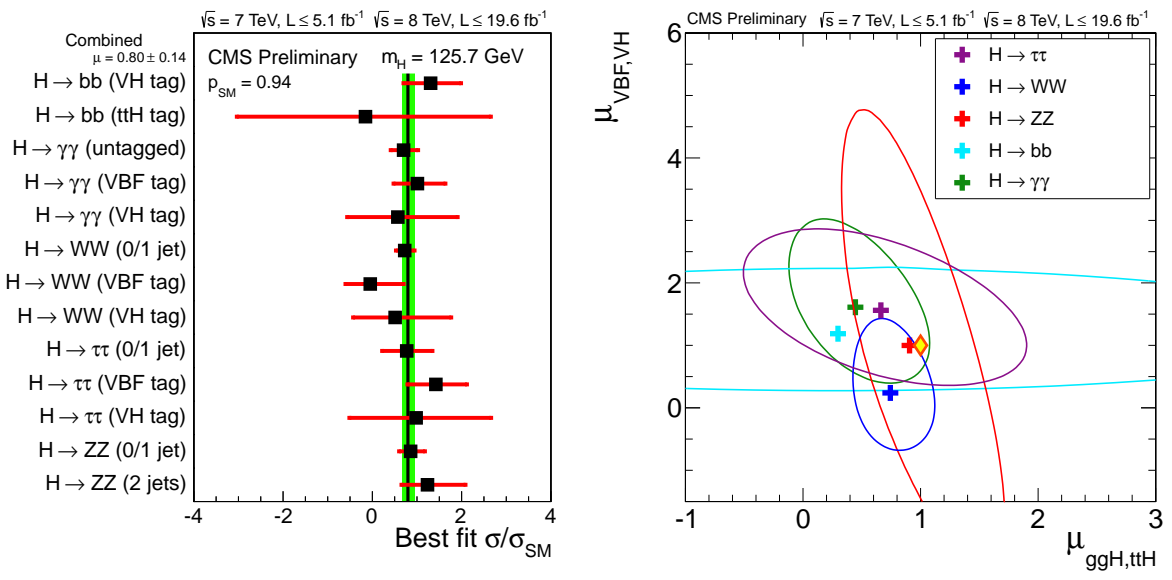

Figure 10: Left: Values of $\sigma / \sigma_{S M}$ for the combination (solid vertical line), for sub-combinations by decay mode and by additional tags targeting a particular production mechanism. The symbol $\sigma / \sigma_{S M}$ denotes the production cross section times the relevant branching fractions, relative to the SM expectation. The horizontal bars indicate the \pm 1 standard deviation uncertainties in the $\sigma / \sigma_{S M}$ values for the individual modes; they include both statistical and systematic uncertainties. Right: The $68 \%$ (solid lines) CL region for the signal strength in the gluon-gluon-fusion-plus- $t \bar{t} H$ and in the VBF-plus-VH production mechanisms, $\mu(g g H+t t H)$ and $\mu(V B F+V H)$, respectively. The different colours show the results obtained by combining data from each of the five analysed decay modes, the diamond at $(1,1)$ indicates the expected values for the SM Higgs boson. 


\subsubsection{Couplings}

The event yield in any (production) $\times$ (decay) mode is assumed to be related to the production cross-section, and the partial and total Higgs boson decay widths via the following equation:

$$
(\sigma \cdot B R)(x \rightarrow H \rightarrow f f)=\frac{\sigma_{x} \cdot \Gamma_{f f}}{\Gamma_{t o t}}
$$

where $\sigma_{x}$ is the production cross section through the initial state $x$ ( $x$ includes gluon-gluon fusion, $V B F, W H$ and $Z H$, and $t \bar{t} H$ ); $\Gamma_{f f}$ is the partial decay width into the final state $f f$ (at present $f$ spans $W, Z, b, t, \gamma$, and $Z \gamma$ ); and $\Gamma_{t o t}$ is the total width of the Higgs boson. To test for possible deviations in the data from the rates expected in the different channels for the SM Higgs boson, we introduce modified couplings, denoted by scale factors $k_{i}$ (where $i$ is the interested particle) and fit the data to these new parameters. Significant deviations of any k from unity would imply new physics beyond the SM Higgs boson hypothesis.

- By using the ratio of event yields in $p p \rightarrow H \rightarrow Z Z$ and $p p \rightarrow H \rightarrow W W$, a test of the custodial symmetry [27][28] has been performed and the result is that the $95 \%$ C.L. interval for $k_{W} / k_{Z}$ is $[0.60,1.40]$.

- By assuming the custodial symmetry is satisfied and assuming that no beyond SM Higgs decay modes are open, the coupling to the fermions $k_{f}$ and bosons $k_{V}$ are tested. The $68 \%$ C.L interval resulting from the fit and the interplay among the different channels is in Fig.11(left). By fixing $k_{f}$ to unity the 1-D likelihood scan has been performed to find the 95\% CL interval of the $k_{V}$ and viceversa. The resulting intervals are $[0.74,1.06]$ for $k_{f}$ and $[0.61,1.33]$ for $k_{V}$.

- If new physics exists, new particles could be produced in loop diagrams, therefore by the study of the processes $g g \rightarrow H$ and $H \rightarrow \gamma \gamma$ can test the SM. The couplings to photons and guons are found to be compatible to the SM ones.

- Tests for variations of the couplings to the fermions with respect to the Yukawa couplings resulted in compatibility with the SM.

- By making several assumptions, one by one tested as reported above, a fit with six free independent coupling $\left(k_{\gamma}, k_{g}, k_{V}, k_{b}, k_{\tau}, k_{t}\right)$ has been performed. The results of the fit for these six parameters, one at a time while profiling the remaining five together with all other nuisance parameters, are listed in Fig.11(right).

\subsubsection{Spin}

In order to study the spin of the new particle, the $H \rightarrow Z Z \rightarrow 4 l$ and $H \rightarrow W W \rightarrow l v l v$ channels have been used. An hypothesis test between the two possibilities $J^{P}=0^{+}$(the SM Higgs boson) and $J^{P}=2_{m}^{+}(g g)$ (a graviton-like boson with minimal couplings produced in gluon fusion) [29]. Fig. 12 shows the post-fit distributions of the test statistic for the background plus signal hypotheses as well as an arrow indicating the value of the test statistic observed in data. The likelihoods for the alternative hypotheses are maximised independently with respect to the nuisance parameters, including the relative signal strengths $\mu_{i}$. The observed value of the test statistic $q$ deviates from the median expected for the $J^{P}=2_{m}^{+}(g g)$ hypothesis by 2.84 standard deviations and is consistent with the median expected for the $J^{P}=0^{+}$hypothesis within 0.34 standard deviations. 

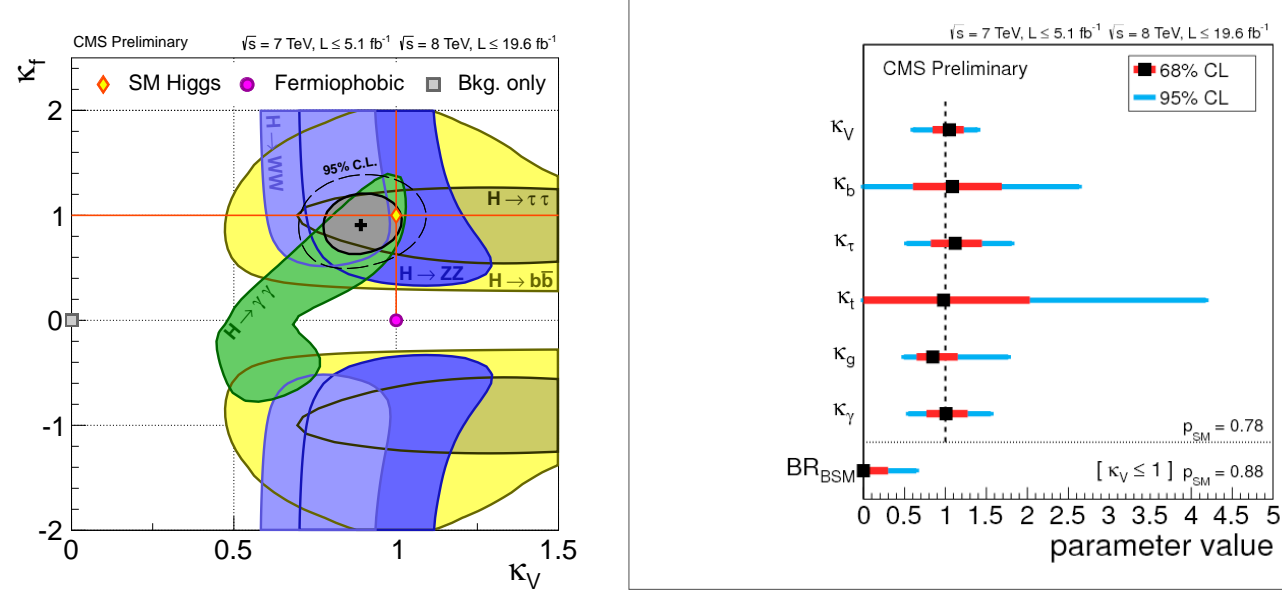

Figure 11: Left: The 68\% C.L. contours for individual channels (coloured swaths) and for the overall combination (solid line) for the $\left(k_{V}, k_{f}\right)$ parameters. The cross indicates the global best-fit values. The thin contour shows the $95 \%$ CL range for the combination. Right: Summary of the results of the six coupling fits.

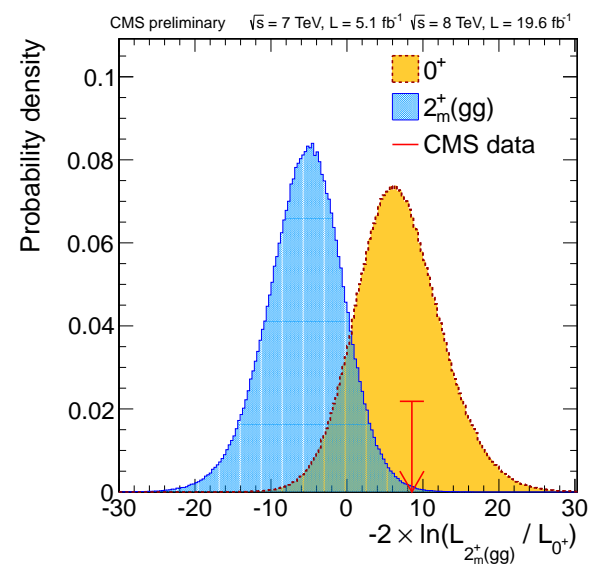

Figure 12: Post-fit model distributions of the test statistic comparing the signal $J^{P}$ hypotheses $0^{+}$and $2_{m}^{+}(g g)$ in the best fit to the data. The observed value is indicated by the arrow and disfavours the $2_{m}^{+}(g g)$ signal hypothesis with a $C L_{s}$ value of $0.60 \%$.

\section{Other analyses}

The Higgs physics program in CMS, is rich of other analyses, besides the ones presented above. Some of them have not been included in the last combination because their results arrived later, or because their sensitivity would not add much to the combination. In the following a short list of some of such results is given.

The search for a Higgs boson in the $Z \gamma$ decay channel has been performed and described in [30]. The upper limit is shown in Fig.13 (left). The sensitivity to the SM Higgs is low for the available integrated luminosity, but this analysis can be important for BSM physics search. For some models 
the $B R(H \rightarrow Z \gamma)$ and $B R(H \rightarrow \gamma \gamma)$ are not correlated, so a combined analysis of the two decay modes can give information on new physics. A $t \bar{t} H$ search, combining the decay channels $H \rightarrow \gamma \gamma$ and $H \rightarrow b \bar{b}$ has been performed, and the upper limit is shown in Fig.13.
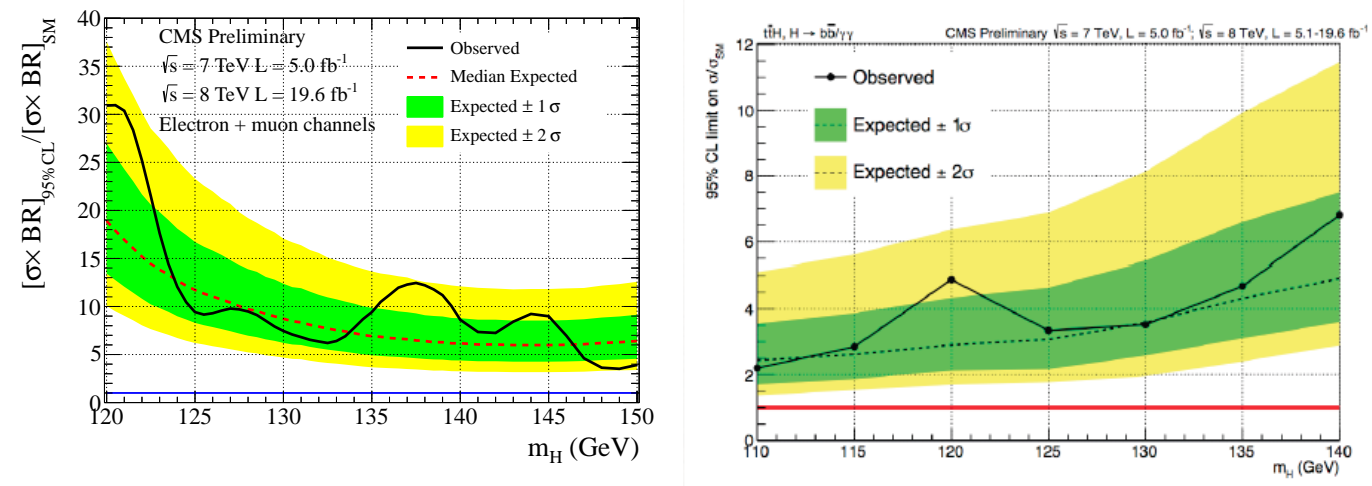

Figure 13: Upper limit as a function of the Higgs mass for the analysis $H \rightarrow Z \gamma$ (left) and $t \bar{t} H$ combining the $H \rightarrow \gamma \gamma$ and $H \rightarrow b \bar{b}$ channels.

New analyses for the decay channel $H \rightarrow b \bar{b}$ have been performed recently [33]. They aimed to select the VBF and $\mathrm{VH}$ production modes respectively. The upper limit resulting from the combination of the two, is shown in Fig.14 (left). The $H \rightarrow W W$ decay channel has been studied recently in the production mode $V H$, with the vector boson decaying to quarks. [34] describes such analysis and the resulting upper limit is shown in 14 (right).
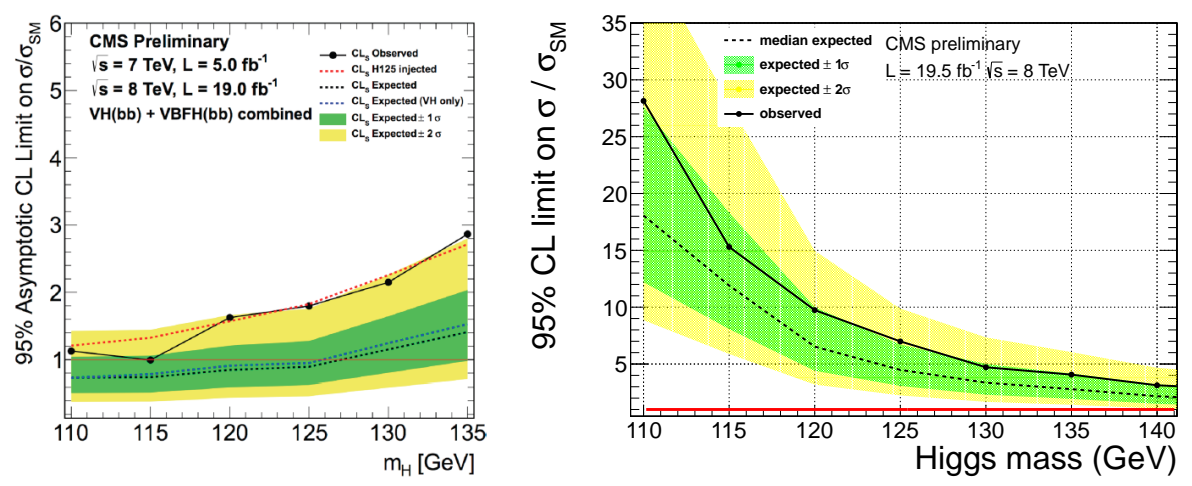

Figure 14: Upper limit as a function of the Higgs mass for the analysis $H \rightarrow b \bar{b}$ combining the analyses targeting the two production mechanisms VBF and VH (left) and for $H \rightarrow W W$ with an analysis selecting the production mode $V H$ with $V \rightarrow j j$.

The high resolution of the $H \rightarrow \gamma \gamma$ channel, have been exploited to test the possibility that the observed signal could be a superposition of two states quasi-degenerate in mass. Also a second Higgs-like boson elsewhere, in the mass range $110<m_{H}<150$ has been searched. No other particle has been found [35].

The analyses $H \rightarrow Z Z H \rightarrow W W$ have been used also to look for another Higgs-like particle at high mass, namely for $m_{H}>200 \mathrm{GeV}$. The result is that no other SM-like Higgs, with SM 
couplings with mass $<1 \mathrm{TeV}$ have been found.

\subsection{Conclusions}

Preliminary results of the analysis of the full dataset collected at LHC in 2011 and 2012 at 7 and $8 \mathrm{TeV}$ centre-of-mass energy and corresponding to integrated luminosities of 5 and 20 $\mathrm{fb}^{-1}$ have been reported. The observed new boson with a measured mass of about $125.7 \pm 0.3$ (stat.) \pm 0.3 (syst.) GeV is found to be consistent with the Standard Model Higgs boson in all investigated properties. Many different searches for beyond the SM Higgs bosons have also been carried out and improved exclusion limits have been obtained.

\section{References}

[1] S.Weinberg, A Model of Leptons, Phys.Rev.Lett. 19 (1967) 1264.

[2] A.Salam Elementary Particle Theory p.367, Almquist and Wiksells, Stocholm,1968.

[3] F.Englert and R. Brout, Broken symmetries and the masses of gauge bosons, Phys.Rev.Lett. 13 (1964) 321.

[4] P. W. Higgs,Broken Symmetry and the mass of gauge vector mesons Phys.Rev.Lett. 13 (1964) 508.

[5] ATLAS Collaboration Observation Phys.Lett. B 716, 1, (2012).

[6] CMS Collaboration Observation Phys.Lett. B 716, 30, (2012).

[7] LHC Higgs Cross Section Working Group et al., Handbook of LHC Higgs cross sections: 1. inclusive observables, (CERN, Geneva, 2011). arXiv:1101.0593.

[8] LHC Higgs Cross SectionWorking Group et al., Handbook of LHC Higgs cross sections: 2. differential distributions, (CERN, Geneva, 2012). arXiv:1201.3084

[9] LHC Higgs Cross SectionWorking Group, Higgs cross sections at 7 and $8 \mathrm{TeV}$, (2012). https://twiki.cern.ch/twiki/bin/view/LHCPhysics/CrossSections.

[10] A. Djouadi, M. Spira, and P. M. Zerwas, Production of Higgs bosons in proton colliders: QCD corrections, Phys. Lett. B 264 (1991) 440, doi:10.1016/0370-2693(91)90375-Z.

[11] NNPDF Collaboration, Impact of heavy quark masses on parton distributions and LHC phenomenology, Nucl. Phys. B 849 (2011) 296, doi:10.1016/j.nuclphysb.2011.03.021, arXiv: 1101.1300

[12] CMS Collaboration The CMS Experiment at the CERN LHC, JINST 3 (2008) S08004.

[13] ATLAS and CMS Collaborations, LHC Higgs Combination Group, Procedure for the LHC Higgs boson search combination in Summer 2011, Technical Report ATL-PHYS-PUB 2011-11, CMS NOTE 2011/005, (2011).

[14] CMS Collaboration, Combined results of searches for the standard model Higgs boson in pp collisions at $\sqrt{s}=7 \mathrm{TeV}$, Phys. Lett. B 710 (2012) 26, doi:10.1016/j.physletb.2012.02.064, arXiv:1202.1488

[15] G. Cowan et al., Asymptotic formulae for likelihood-based tests of new physics, Eur. Phys. J. C 71 (2011) 1, doi:10.1140/epjc/s10052-011-1554-0, arXiv:1007.1727.

[16] L. Moneta et al., The RooStats project, in 13th International Workshop on Advanced Computing and Analysis Techniques in Physics Research (ACAT2010). SISSA, 2010. arXiv:1009.1003. PoS(ACAT2010)057. 
[17] CMS Colaboration, CMS-PAS-HIG-13-005 Measurement of the properties of the new boson with a mass near $125 \mathrm{GeV}$

[18] CMS-PAS-HIG-13-001 Search for the standard model Higgs boson in the $H \rightarrow \gamma \gamma$ channel in pp collisions at $\sqrt{s}=7$ and $8 \mathrm{TeV}$

[19] CMS-PAS-HIG-13-002 Search for the standard model Higgs boson in the $H \rightarrow$ ZZ channel in pp collisions at $\sqrt{s}=7$ and $8 \mathrm{TeV}$

[20] CMS-PAS-HIG-13-003 Search for the standard model Higgs boson in the $H \rightarrow W W \rightarrow l v l v$ channel in pp collisions at $\sqrt{s}=7$ and $8 \mathrm{TeV}$

[21] CMS-PAS-HIG-12-042 Evidence for a particle decaying into $W^{+} W^{-}$in the fully leptonic final state in a standard model Higgs bosons search in pp collisions at $\sqrt{s}=8 \mathrm{TeV}$

[22] CMS-PAS-HIG-13-009 Search for the standard model Higgs boson in the $W H \rightarrow W W W \rightarrow 3 l 3 v$ channel in pp collisions at $\sqrt{s}=7$ and $8 \mathrm{TeV}$

[23] CMS-PAS-HIG-13-004 Search for the standard model Higgs boson decaying to tau pairs in proton-proton collisions at $\sqrt{\mathrm{s}}=7$ and $8 \mathrm{TeV}$.

[24] CMS-PAS-HIG-12-053 Search for the standard model Higgs boson decaying to tau pairs produced in association with aWor Z boson with the CMS experiment in pp collisions at $\sqrt{s}=7$ and $8 \mathrm{TeV}$.

[25] CMS-PAS-HIG-12-044 Search for standard model Higgs bosons produced in association with W or Z bosons, and decaying to bottom quarks

[26] arXiv:1303.0763, JHEP Search for the standard model Higgs boson produced in association with a top-quark pair in pp collisions at the LHC

[27] M. Veltman, Limit on mass differences in theWeinberg model, Nucl. Phys. B 123 (1997) 89, doi:10.1016/0550-3213(77)90342-X.

[28] P. Sikivie et al., Isospin breaking in technicolor models, Nucl. Phys. B 173 (1980) 189, doi:10.1016/0550-3213(80)90214-X.

[29] S. Bolognesi et al., On the spin and parity of a single-produced resonance at the LHC, Phys. Rev. D 86 (2012) 095031, doi:10.1103/PhysRevD.86.095031, arXiv:1208.4018.

[30] CMS-PAS-HIG-13-006 Search for the standard model Higgs boson in the Z boson plus a photon channel in pp collisions at $\sqrt{s}=7$ and $8 \mathrm{TeV}$.

[31] CMS-PAS-HIG-13-015 Search for ttH production in events where H decays to photons at $8 \mathrm{TeV}$ collisions.

[32] CMS-PAS-HIG-13-011 Higgs to $b \bar{b}$ in the VBF channel.

[33] CMS-PAS-HIG-13-012 Search for the standard model Higgs boson produced in association with W or $Z$ bosons, and decaying to bottom quarks for LHCP

[34] CMS-PAS-HIG-13-017 VH with $H \rightarrow W W \rightarrow l v l v$ and $V \rightarrow j j$

[35] CMS-PAS-HIG-13-016 Properties of the observed Higgs-like resonance using the diphoton channel 